\title{
AVALIAÇÃO DE TRÊS TIPOS DE ESTRUTURA DE COLCHÃO E TRÊS NÍVEIS DE RESINA FENÓLICA NA PRODUÇÃO DE CHAPAS DE PARTÍCULAS ORIENTADAS - OSB ${ }^{1}$
}

\author{
Fernando Nunes Gouveia ${ }^{2}$, Benedito Rocha Vital ${ }^{3}$ e Marcos Antônio Eduardo Santana ${ }^{4}$
}

\begin{abstract}
RESUMO - O objetivo deste trabalho foi avaliar a influência da estrutura do colchão e de diferentes concentrações de adesivo nas propriedades de chapas de partículas orientadas (strands), contendo 50\% de partículas de Eucalyptus grandis e $50 \%$ de Pinus elliottii. Foram avaliados três tipos de estruturas. Em uma das estruturas as partículas foram distribuídas em camadas indistintas. Uma segunda estrutura foi constituída de $50 \%$ de partículas de eucalipto no miolo do colchão e $25 \%$ de pinus em cada face. A terceira estrutura foi formada por $50 \%$ de madeira de pinus no miolo e $25 \%$ de eucalipto nas faces. Cada uma dessas estruturas foi aglutinada com 4,6 ou $8 \%$ de sólidos resinosos em relação ao peso seco das partículas de um adesivo fenólico com viscosidade de $350 \mathrm{cps}$, pH 12,15 e $47,63 \%$ de sólidos. Para os nove tratamentos foram feitas três repetições, totalizando 27 chapas. As chapas foram submetidas aos testes de flexão estática, ligação interna, arranque de parafuso, inchamento em espessura e absorção de água, obedecendo aos critérios da norma ASTM D 1037 - 91. De modo geral os painéis feitos com 8\% de adesivo apresentaram melhores resultados que os demais. Para a maioria das propriedades mecânicas, inclusive para os painéis fabricados com $4 \%$ de resina, obtiveram-se resultados superiores àqueles estabelecidos pela Norma CSA O437-93. Contudo, nenhum tratamento produziu chapas com inchamento em espessura inferior ao valor máximo permitido por aquela norma. Não se verificou diferença estatística, ocasionada pela estrutura de colchão, entre todas as propriedades avaliadas.
\end{abstract}

Palavras-chave: $\quad$ OSB, eucalipto, pinus, resina fenólica e colchão.

\section{EVALUATION OF THREE MAT STRUCTURES AND THREE PHENOLIC RESIN LEVELS FOR ORIENTED STRAND BOARD PRODUCTION}

\begin{abstract}
This work aimed to evaluate the influence of mat structure and different adhesive contents on the properties of oriented strand boards (OSB) made of 50\% Eucalyptus grandis and $50 \%$ Pinus elliottii strands. Three types of structure were employed: the first consisted of indistinct layers, the second of boards made of 50\% eucalypt strands in the core and 25\% pine on each face, and the third structure containing eucalypt (25\%) in each face, and core Pinus (50\%). A total of 4\%, 6\% or $8 \%$ of phenolic resin, based on the oven-dry weight of the particles, was applied on all the structures. Resin total solids were equal to $47.63 \%, \mathrm{pH} 12.15$ and viscosity 350cps. A total of 27 boards, including three replications, were manufactured and tested for static bending (MOR and MOE), internal board, screw withdrawal, thickness swelling and water absorption, according to ASTM D 1037 - 91 procedures. In general, boards using 8\% of resin obtained the best results. Most of the treatments, including the boards containing 4\% adhesive, achieved mechanical resistances higher than those established under CSA-O437-93 standard. None of the treatments was sufficiently good for thickness swelling. Mat structure had no effect on board properties.
\end{abstract}

Key words: $\quad$ OSB, Eucalyptus, Pinus, phenolic resin and mat.

1 Recebido para publicação em 18.12.2001.

Aceito para publicação em 12.5.2003.

2 Engenheiro Florestal, Mestre em Ciências Florestais, <fgouveia@email.com.br >. ${ }^{3}$ Engenheiro Florestal, Ph.D. Professor do Departamento de Engenharia Florestal da Universidade Federal de Viçosa - UFV, 36571-000 Viçosa-MG, <bvital@ufv.br>. ${ }^{4}$ Químico, Ph.D. Laboratório de Produtos Florestais/IBAMA, SAIN Av. L4 Lote 04, 70818-900 Brasília-DF, $<$ marcos@csr-lpf.ibama.gov.br>. 


\section{INTRODUÇÃO}

As chapas de partículas orientadas, comercialmente denominadas de OSB - Oriented Strand Boards, são painéis indicados principalmente para fabricação de embalagens e emprego na construção civil. O OSB difere das chapas de madeira aglomerada tradicional por utilizar partículas longas e finas, parcialmente orientadas em uma determinada direção, e por ter boa resistência mecânica e boa estabilidade dimensional, competindo diretamente com o compensado (Mendes et al., 2000). Pode ter uma, três ou mais camadas, formando uma estrutura semelhante à do compensado. A orientação das partículas permite a obtenção de propriedades mecânicas que se assemelham às da madeira maciça (Shaler, 1991). As chapas de OSB, comparadas com as de compensado, são menos exigentes quanto a vários índices de qualidade da madeira. Por isto é possível fabricar tais chapas com preço competitivo, e em alguns países como os Estados Unidos e o Canadá o OSB conquistou parte do mercado antes dominado exclusivamente pelos compensados, principalmente o de embalagens e de construção civil (Gouveia et al., 2000).

No mercado internacional, a demanda potencial por esse tipo de painel é muito grande, uma vez que nos Estados Unidos a construção de novas habitações emprega cerca de um terço do consumo anual de madeira serrada e painéis de madeira do mercado (Howard, 2000). Em outros países como Alemanha, França e China também foi observado crescimento significativo no consumo dos diversos tipos de painéis de madeira reconstituída (Juvenal \& Mattos, 2002).

No Brasil, em 2001, a produção de painéis de madeira reconstituída (aglomerado, MDF e chapa de fibra) foi igual a $2.976 \mathrm{~m}^{3}$. Naquele ano, a produção brasileira destinou-se principalmente ao mercado interno, uma vez que foram exportados apenas $193.000 \mathrm{~m}^{3}$ destes painéis, sendo $181.000 \mathrm{~m}^{3}$ de chapas de fibra. A primeira planta industrial para produção de OSB no Brasil foi concluída no final de 2001 e teve a capacidade estimada em $200.000 \mathrm{~m}^{3}$ para o ano de 2002 (Juvenal \& Mattos, 2002).

O início da produção comercial de OSB ocorreu na região dos Grandes Lagos, Canadá, utilizando principalmente a madeira de bétula (Populus spp.). Em seguida, a indústria se expandiu por quase toda a América do Norte e outros continentes. Outras espécies foram e continuam sendo pesquisadas para utilização na indústria de OSB como matéria-prima, no entanto este é um desafio

R. Árvore, Viçosa-MG, v.27, n.3, p.365-370, 2003 constante, principalmente quando se busca o emprego de madeiras de folhosas (Wang \& Winistorfer, 2000).

Segundo esses autores, a utilização de diferentes espécies e misturas na fabricação de chapas de composição é descrita em diversos artigos científicos. A premissa básica de todas essas pesquisas tem sido o fato de as chapas de partículas orientadas poderem ser produzidas com outras espécies, misturas de espécies ou diferentes estruturas de camadas.

O objetivo deste trabalho foi a avaliação das propriedades físicas e mecânicas de chapas de partículas orientadas - OSB, produzidas a partir de três tipos de estrutura de colchão e de três taxas de aplicação de resina, empregando partículas finas e longas (strands) obtidas a partir de madeiras de Eucalyptus grandis e Pinus elliottii.

\section{MATERIAL E MÉTODOS}

As chapas foram produzidas com misturas de madeiras de 18 árvores de Eucalyptus grandis W. Hill ex Maiden e três árvores de Pinus elliottii Engelm., cujas árvores foram obtidas, respectivamente, em plantios com 7 anos de idade, na fazenda Cachoeirinha, no município de Viçosa-MG, pertencente à Universidade Federal de Viçosa (UFV), e em plantios com 30 anos de idade localizados no campus da UFV.

Os valores médios de densidade básica das madeiras de Eucalyptus grandis e Pinus elliottii foram iguais a 0,52 e $0,43 \mathrm{~g} / \mathrm{cm}^{3}$, respectivamente.

As árvores foram secionadas em número variável de toras, dependendo da altura da árvore, as quais foram cortadas com $4 \mathrm{~m}$ de comprimento e, posteriormente, em toretes de $1 \mathrm{~m}$. Os toretes foram desdobrados em tábuas, com aproximadamente $80 \mathrm{~cm}$ de comprimento, $12 \mathrm{~cm}$ de largura e 2,0 cm de espessura. Posteriormente, as tábuas foram reduzidas a peças com mesma largura e espessura e com $20 \mathrm{~cm}$ de comprimento, as quais foram armazenadas em tanques com água, durante pelo menos duas semanas, até estarem completamente saturadas.

Depois de saturadas, as peças de eucalipto foram aquecidas em água fervente, durante $1 \mathrm{~h}$. Este processo foi realizado com o intuito de favorecer o corte da madeira, proporcionando assim partículas de melhor qualidade. Em seguida as peças de madeira de eucalipto e pinus foram processadas em um picador Bezner, gerando partículas que foram espalhadas sob lonas para secarem ao ar. Todo o material foi levado a um sistema 
de peneiras classificador de partículas, por gravidade. Uma malha de $24 \mathrm{~mm}$ e outra de $14 \mathrm{~mm}$ foram utilizadas, em conjunto, para separar as partículas. O material não retido nas telas foi desprezado, e as partículas classificadas foram submetidas à secagem, em estufa de laboratório com ventilação forçada a $95{ }^{\circ} \mathrm{C}$, até o teor de umidade de $3 \%$. As dimensões das partículas foram, aproximadamente, $10 \mathrm{~cm}$ de comprimento, $2 \mathrm{~cm}$ de largura e $0,52 \mathrm{~mm}$ de espessura.

O adesivo utilizado foi o CASCOPHEN IV-80 da Alba Química, uma resina fenólica com $350 \mathrm{cps}$ de viscosidade, $\mathrm{pH} 12,15$ e $47,63 \%$ de teor de sólidos resinosos. Foi adicionado $1 \%$ de emulsão de parafina às chapas em relação ao peso seco das partículas, com o objetivo de retardar a absorção de água. A emulsão foi preparada no próprio laboratório, obedecendo à seguinte proporção: 380 partes de parafina, 360 partes de água, 40 partes de $\mathrm{NH}_{4} \mathrm{OH} 22 \%$ e 40 partes de ácido esteárico.

A proporção entre as madeiras de Eucalyptus grandis e Pinus elliottii foi fixada em 50\%. As chapas foram confeccionadas nas dimensões de $55 \times 55 \times 1,3 \mathrm{~cm}$, com densidade nominal de $0,7 \mathrm{~g} / \mathrm{cm}^{3}$, e três diferentes teores de sólidos de resina em relação ao peso seco das partículas, 4,6 ou 8\%. O adesivo foi aplicado por meio de um misturador mecânico tipo DRAIS FSP 80. O colchão, composto de três camadas cruzadas de partículas (face: $25 \%$, miolo: $50 \%$, face: $25 \%$ ), foi formado manualmente e as partículas foram orientadas mecanicamente através de um sistema de canaletas. O colchão foi prensado em uma prensa hidráulica SIMI com aquecimento elétrico, durante 8 minutos, a uma temperatura média de $147,38^{\circ} \mathrm{C}$ e tempo médio de fechamento de prensa de 23 '. Teve-se como meta empregar uma taxa de compressão igual a 1,50, o que, considerando a densidade média da mistura contendo $50 \%$ de cada espécie igual a $0,48 \mathrm{~g} / \mathrm{cm}^{3}$, resultaria em chapas com densidade igual a $0,72 \mathrm{~g} / \mathrm{cm}^{3}$. Esta taxa de compressão é superior a 1,30, que é o valor mínimo recomendado por Maloney (1993) para fabricação de chapas com boa resistência mecânica.

Para cada tipo de mistura foram avaliadas três quantidades de adesivo, já previamente descritas e indicadas no Quadro 1. Assim, foram prensadas 27 chapas, com três composições do colchão, três teores de adesivo e três repetições.

Após a climatização das chapas, foram retirados quatro corpos-de-prova, medindo aproximadamente de $7,6 \times 35 \mathrm{~cm}$, para determinação do módulo de ruptura
(MOR) e de elasticidade (MOE). Adicionalmente, foram produzidos três corpos-de-prova por chapa, nas dimensões nominais de $5 \times 5 \mathrm{~cm}$, para o ensaio de tração perpendicular ao plano da chapa (ligação interna). Para $o$ ensaio de arranque de parafuso foram confeccionados dois corpos-de-prova com dimensões aproximadas de $7,6 \times 15 \mathrm{~cm}$.

As propriedades mecânicas foram determinadas segundo o procedimento descrito na Norma ASTM D 1037-91 (ASTM, 1991).

Para determinação da densidade e do teor de umidade foram utilizados dois corpos-de-prova, nas dimensões de 7,6 × 7,6 cm. Para obtenção do volume a ser utilizado no cálculo da densidade foi utilizado o método de medição direta, medindo-se as três dimensões das peças com um paquímetro digital. $\mathrm{O}$ teor de umidade foi calculado na base seca.

Para os ensaios de inchamento em espessura e absorção de água foram retirados dois corpos-de-prova nas dimensões de 7,6×30,5 cm, de cada chapa. Após a sua climatização os corpos-de-prova foram pesados, e com o auxílio de um gabarito determinou-se a espessura em quatro pontos marcados, distantes $2,5 \mathrm{~cm}$ das bordas dos corpos-de-prova. Os corpos-de-prova foram, então, mergulhados em um recipiente com água destilada, e após 2 e 24 horas foram refeitas as medições e pesagens.

Os dados foram submetidos à análise de variância (ANOVA) e ao teste de Tukey, a 5\% de probabilidade, com o intuito de avaliar o efeito das diferentes estruturas de colchão e taxas de adesivo. A análise foi desenvolvida com o auxílio do programa SPSS (1999). Os resultados obtidos foram também comparados com aqueles estabelecidos pela Norma CSA O437-93 (1993).

Quadro 1 - Delineamento do experimento Table 1 - Experimental design

\begin{tabular}{|c|c|c|c|c|c|}
\hline $\begin{array}{c}\text { Composição } \\
\text { do Colchão* }\end{array}$ & $\begin{array}{c}\text { Estrutura } \\
\text { do } \\
\text { Colchão }\end{array}$ & \multicolumn{3}{|c|}{ Teor de Adesivo (\%) } & Repetições \\
\hline MIS & $1: 2: 1$ & 8 & 6 & 4 & 3 \\
EPE & $1: 2: 1$ & 8 & 6 & 4 & 3 \\
PEP & $1: 2: 1$ & 8 & 6 & 4 & 3 \\
\hline
\end{tabular}

* MIS - Mistura de partículas de pinus e eucalipto; EPE - partículas de eucalipto nas faces e de pinus na camada central; e PEP partículas de pinus nas faces e de eucalipto na camada central. 


\section{RESULTADOS E DISCUSSÃO}

No Quadro 2 estão listados os valores médios da densidade básica e taxa de compressão para cada tratamento. As chapas apresentaram valores de densidade um pouco superiores ao desejado, que é de a $0,70 \mathrm{~g} / \mathrm{cm}^{3}$, porém não houve diferença estatística entre eles. Assim, a taxa de compressão, obtida ao dividir a densidade das chapas pela média das densidades das madeiras, foi superior a 1,30 , que é o valor mínimo recomendado por Maloney (1993) para fabricação de chapas com boa resistência mecânica.

Os valores médios de resistência à flexão estática, de ligação interna e de resistência ao arranque de parafusos estão no Quadro 3.

Quadro 2 - Valores médios da densidade e taxa de compressão*

Table 2 - Average values for board density and compression ratio

\begin{tabular}{|c|c|c|}
\hline $\begin{array}{c}\text { Composição do } \\
\text { Colchão }\end{array}$ & $\begin{array}{c}\text { Densidade** } \\
\left(\mathrm{g} / \mathrm{cm}^{3}\right)\end{array}$ & $\begin{array}{c}\text { Taxa de } \\
\text { Compressão }\end{array}$ \\
\hline MIS & $0,73 \mathrm{a}$ & 1,54 \\
EPE & $0,72 \mathrm{a}$ & 1,51 \\
PEP & $0,75 \mathrm{a}$ & 1,59 \\
\hline
\end{tabular}

* Valores em uma mesma coluna, seguidos por uma mesma letra, são idênticos estatisticamente.

** Massa/volume a $12 \%$ de umidade.
Não foi constatada diferença significativa na resistência à flexão estática, na ligação interna e na resistência ao arranque de parafusos provocada pela estrutura das chapas. Contudo, considerando o teor de adesivo dentro de cada estrutura, observou-se, com algumas exceções, que a redução no teor de adesivo ocasionou diminuição em todas as propriedades. Entretanto, diferenças significativas foram observadas apenas para resistência à flexão na direção perpendicular à orientação partículas, para ligação interna e para a resistência ao arranque de parafuso, pois as chapas fabricadas com $4 \%$ de adesivo apresentaram valores médio inferiores.

Apenas para as chapas fabricadas com estrutura indistinta (MIS) e 4\% de adesivo foram obtidos valores médios de resistência ao arranque de parafuso inferior ao limite estabelecido pela Norma CSA O437-93 (1993). Para os demais tratamentos obtiveram-se propriedades com valores superiores ao mínimo estabelecido pela norma.

Um detalhe interessante foi observado no rompimento dos corpos-de-prova nos testes de ligação interna dos dois últimos tratamentos, PEP e EPE. Nas chapas produzidas com madeira de eucalipto nas camadas superficiais (EPE), $81 \%$ dos corpos-de-prova se romperam em uma das faces, uma vez que nas chapas produzidas com eucalipto no miolo (PEP) 59\% amostras se romperam na camada interna. Este comportamento pode ser explicado pela diferença entre as espécies, pois como a madeira de

Quadro 3 - Valores médios dos ensaios de flexão estática (MOE, MOR), ligação interna e arranque de parafusos* Table 3 - Average values for the static bending test (MOE, MOR), internal bond and screw withdrawal

\begin{tabular}{|c|c|c|c|c|c|c|c|}
\hline \multicolumn{2}{|c|}{ Tratamento } & \multicolumn{2}{|c|}{$\begin{array}{c}\text { Flexão Paralela } \\
(\mathrm{MPa})\end{array}$} & \multicolumn{2}{|c|}{$\begin{array}{c}\text { Flexão Perpendicular } \\
(\mathrm{MPa})\end{array}$} & \multirow{2}{*}{$\begin{array}{l}\text { Ligação Interna } \\
(\mathrm{MPa})\end{array}$} & \multirow{2}{*}{$\begin{array}{l}\text { Arranque de } \\
\text { Parafuso } \\
\text { (N) }\end{array}$} \\
\hline Composição & Adesivo & MOR & MOE & MOR & MOE & & \\
\hline \multirow{3}{*}{ MIS } & $8 \%-\mathrm{T} 1$ & $43,89 a$ & $6.838,57 \mathrm{a}$ & $39,80 \mathrm{a}$ & $4.732,32 \mathrm{a}$ & $0,706 a$ & $2.185 \mathrm{a}$ \\
\hline & $6 \%-\mathrm{T} 2$ & $41,31 \mathrm{a}$ & $5.500,73 a$ & $37,65 \mathrm{a}$ & $4.546,16 \mathrm{a}$ & $0,551 \mathrm{a}$ & $2.001 \mathrm{a}$ \\
\hline & $4 \%-\mathrm{T} 3$ & $36,89 \mathrm{a}$ & $6.159,55 \mathrm{a}$ & $30,96 b$ & $4.213,73 a$ & $0,457 \mathrm{~b}$ & $1.383 b$ \\
\hline \multirow{3}{*}{ EPE } & $8 \%-\mathrm{T} 4$ & $43,19 \mathrm{a}$ & $6.771,22 \mathrm{a}$ & $35,46 a$ & $4.875,02 \mathrm{a}$ & $0,637 \mathrm{a}$ & $2.177 \mathrm{a}$ \\
\hline & $6 \%-\mathrm{T} 5$ & $37,61 \mathrm{a}$ & $6.159,07 \mathrm{a}$ & $40,28 \mathrm{a}$ & $5.316,33 \mathrm{a}$ & $0,705 \mathrm{a}$ & $2.403 a$ \\
\hline & $4 \%-\mathrm{T} 6$ & $33,66 \mathrm{a}$ & $6.077,45 \mathrm{a}$ & $28,37 \mathrm{~b}$ & $4.139,82 \mathrm{a}$ & $0,442 b$ & $1.654 \mathrm{~b}$ \\
\hline \multirow{3}{*}{ PEP } & $8 \%-\mathrm{T} 7$ & $49,29 a$ & $6.717,14 a$ & $44,31 \mathrm{a}$ & $5.601,92 a$ & $0,659 \mathrm{a}$ & $2.025 \mathrm{a}$ \\
\hline & $6 \%-\mathrm{T} 8$ & $44,29 \mathrm{a}$ & $6.536,05 \mathrm{a}$ & $39,41 \mathrm{a}$ & $5.125,06 a$ & $0,573 a$ & $2.200 \mathrm{a}$ \\
\hline & $4 \%-\mathrm{T} 9$ & $41,75 \mathrm{a}$ & $6.004,81 \mathrm{a}$ & $34,49 b$ & $4.479,99 \mathrm{a}$ & $0,552 b$ & $1.677 \mathrm{~b}$ \\
\hline
\end{tabular}

Valores mínimos exigidos pela norma CSA O437 para OSB da classe 0-2: MOR //: $29 \mathrm{MPa}$; MOR $\perp:$ 12,4 MPa; MOE//: $5500 \mathrm{MPa}$; MOE? $1500 \mathrm{MPa}$; LI: 0,345 MPa, AP: $1498 \mathrm{~N}$. Análise estatística em função da taxa de adesivo.

* Valores em uma mesma coluna, seguidos por uma mesma letra, são idênticos estatisticamente. 
E. grandis é mais densa que a de P.elliottii o volume de material é menor e, conseqüentemente, o contato entre as partículas também o é, daí a fragilidade das camadas onde se encontra a madeira de eucalipto. Outra possibilidade é a menor interatividade entre a madeira de eucalipto e o adesivo.

Os resultados dos testes de absorção de água e inchamento em espessura após imersão em água por $2 \mathrm{e}$ 24 horas estão no Quadro 4.

Analisando apenas a estrutura dos colchões, observou-se que não houve diferenças significativas. Contudo, os melhores resultados numéricos para absorção de água em 24 horas foram obtidos pelos tratamentos PEP e MIS, o que indica que painéis feitos com madeira de eucalipto nas camadas superficiais absorvem mais água do que aqueles fabricados com madeira de pinus ou a mistura destas duas espécies.

Quadro 4 - Valores médios para absorção de água e inchamento em espessura*

Table 4 - Average values for water absorption and thickness swelling

\begin{tabular}{|c|l|c|l|r|c|}
\hline \multicolumn{2}{|c|}{ Tratamento } & \multicolumn{2}{c|}{$\begin{array}{c}\text { Absorção de Água } \\
(\%)\end{array}$} & \multicolumn{2}{c|}{$\begin{array}{c}\text { Inchamento em } \\
\text { Espessura (\%) }\end{array}$} \\
\hline Comp. & Ades. & $2 \mathrm{~h}$ & \multicolumn{1}{c|}{$24 \mathrm{~h}$} & \multicolumn{1}{c|}{$2 \mathrm{~h}$} & $24 \mathrm{~h}$ \\
\hline \multirow{3}{*}{ MIS } & $8 \%$ - T1 & $6,02 \mathrm{a}$ & $22,33 \mathrm{a}$ & $7,94 \mathrm{a}$ & $17,97 \mathrm{a}$ \\
& $6 \%-\mathrm{T} 2$ & $6,92 \mathrm{a}$ & $37,53 \mathrm{ab}$ & $10,03 \mathrm{a}$ & $26,09 \mathrm{~b}$ \\
& $4 \%-\mathrm{T} 3$ & $15,13 \mathrm{a}$ & $53,07 \mathrm{~b}$ & $17,92 \mathrm{~b}$ & $34,93 \mathrm{c}$ \\
\hline \multirow{4}{*}{ EPE } & $8 \%-\mathrm{T} 4$ & $8,18 \mathrm{a}$ & $42,08 \mathrm{a}$ & $8,83 \mathrm{a}$ & $24,38 \mathrm{a}$ \\
& $6 \%-\mathrm{T} 5$ & $11,26 \mathrm{a}$ & $43,95 \mathrm{ab}$ & $10,01 \mathrm{a}$ & $24,04 \mathrm{~b}$ \\
& $4 \%-\mathrm{T} 6$ & $12,40 \mathrm{a}$ & $56,58 \mathrm{~b}$ & $16,18 \mathrm{~b}$ & $36,66 \mathrm{c}$ \\
\hline \multirow{4}{*}{ PEP } & $8 \%-\mathrm{T} 7$ & $5,26 \mathrm{a}$ & $24,45 \mathrm{a}$ & $7,34 \mathrm{a}$ & $20,98 \mathrm{a}$ \\
& $6 \%-\mathrm{T} 8$ & $6,83 \mathrm{a}$ & $32,99 \mathrm{ab}$ & $10,38 \mathrm{a}$ & $27,30 \mathrm{~b}$ \\
& $4 \%-\mathrm{T} 9$ & $5,71 \mathrm{a}$ & $35,24 \mathrm{~b}$ & $11,88 \mathrm{~b}$ & $33,34 \mathrm{c}$ \\
\hline
\end{tabular}

Valor máximo permitido pela norma CSA O437 para IE (24 h) de chapas da classe O-2 e espessura superior a $12,7 \mathrm{~mm}$ : $10 \%$.. Análise estatística em função da taxa de adesivo.

* Valores em uma mesma coluna, seguidos por uma mesma letra, são idênticos estatisticamente.

As outras propriedades físicas não sofreram influência da estrutura do colchão. Entretanto, ao analisar exclusivamente o efeito teor do adesivo, é possível verificar a influência deste em duas das três propriedades físicas analisadas. No teste de absorção de água em 24 horas, os melhores tratamentos foram os das chapas produzidas com taxas de 8 e $6 \%$, tendo os mesmos resultados sido observados no teste de inchamento em espessura em 2 horas. Já no teste de inchamento em 24 horas os melhores resultados foram obtidos exclusivamente por aquelas chapas produzidas com $8 \%$ de adesivo fenólico.

Analisando a interação entre as variáveis estrutura de colchão e a taxa de adesivo, constata-se que a única propriedade a apresentar diferença estatística significativa foi o inchamento em espessura em 24 horas. Deste modo, pode-se concluir que para as chapas produzidas ao misturar as espécies os melhores resultados são obtidos ao utilizar 8\% de adesivo; para painéis fabricados com as faces de partículas de eucalipto, as melhores chapas foram fabricadas ao utilizar 6 ou $8 \%$. Para as chapas produzidas com pinus nas faces, devem ser aplicados maiores teores de adesivo, uma vez que os melhores resultados foram obtidos com $8 \%$ de resina.

A norma canadense CSA 0437-93 somente avalia o teste de inchamento em espessura em 24 horas, distinguindo as chapas com espessura superior a $12,7 \mathrm{~mm}$ daquelas com menor espessura. Todas as chapas produzidas neste experimento apresentaram valores de inchamento superiores ao valor máximo permitido pela norma.

Como sugestões para trabalhos futuros deve-se:

- Fabricar e avaliar painéis OSB com densidade nominal, menor ou igual a $0,60 \mathrm{~g} / \mathrm{cm}^{3}$.

- Avaliar a influência de outras formulações de emulsão de parafina, com o objetivo de melhorar a estabilidade dimensional.

\section{CONCLUSÕES}

Os melhores resultados foram obtidos nos painéis fabricados com 6 e $8 \%$ de fenol-formaldeído.

A estrutura das chapas não ocasionou diferença significativa na resistência à flexão estática, na ligação interna e na resistência do arranque de parafusos.

\section{REFERÊNCIAS BIBLIOGRÁFICAS}

\author{
AMERICAN SOCIETY OF TESTING MATERIALS - \\ ASTM. Standard Test Methods for Evaluating \\ Properties of Wood-base Fiber and Particle Panel \\ Materials. ASTM D 1037 - 91. Philadelphia: Annual \\ Book of ASTM Standards. 1991. (v. 04.09 wood)
}

CANADIAN STANDARDS ASSOCIATION - CSA. Standards on OSB and waferboard - Forest Products. Rexdale: O437 Series-93, 1993.

R. Árvore, Viçosa-MG, v.27, n.3, p.365-370, 2003 
JUVENAL, T. L.; MATTOS, R. L. G. Paineis de madeira reconstituída. Brasília: BNDES, 2002. 21 p.

GOUVEIA, F. N.; SANTANA, M. A. E.; SOUZA, M. R. Utilização da madeira de Eucalyptus grandis W. Hill ex Maiden e Eucalyptus urophylla S. T. Blake na fabricação de chapas de partículas orientadas (OSB) e não orientadas.

Revista Árvore., v. 24. n. 1. p. 7-12, 2000.

HOWARD, J. L. U. S. Forest products annual market review and prospects, $1999-2000$. Research Note. FPL RNP - 0278. Madison: Department of Agriculture, Forest Service, Forest Products Laboratory. 2000. 5 p.
MENDES, L. M. et al. Avaliação do sistema de orientação de partículas na produção de painéis OSB (Oriented strand board). Cerne, v. 6, n. 1, p. 1-8, 2000.

SHALER, S. M. Comparing two measures of flake alignment. Wood Science and Technology, v. 26. p. 53-61, 1991.

STATISTICAL PACKAGE FOR THE SOCIAL SCIENCES - SPSS. Chicago, 1999. (v. 10.0 SPSS)

WANG, S.; WINISTORFER, P. M. The effect of species and species distribution on the layer characteristics of OSB. Forest Products Journal, v. 50, n. 4, p. 37-48, 2000. 